\title{
El uso de los gestos en el discurso docente durante la planificación del juego en el jardín de infantes
}

\author{
Maia Julieta Migdalek \& Celia Rosemberg \\ Universidad de Buenos Aires y \\ Consejo Nacional de Investigaciones Científicas y Técnicas, Buenos Aires, Argentina
}

(Artículo recibido el 28 de febrero de 2012; versión final recibida el 15 de agosto de 2012)

\begin{abstract}
Desde la teoría sociocultural del desarrollo humano (Vigotsky, 2009; Bruner, 1986) se concibe a la interacción situada como el espacio privilegiado para el desarrollo del lenguaje. Dado que en ella las formas lingüísticas están imbricadas con otros sistemas semióticos, en el trabajo se analiza el uso de los gestos de la maestra en la planificación del juego en una sala de jardín de infantes de niños de 4 años. El análisis sigue una lógica cualitativa que articula el Método Comparativo Constante (Strauss y Corbin, 1991) con el empleo heurístico de conceptos del Análisis de la Conversación (Goodwin 2000; Tusón, 1995), el microanálisis discursivo (Gumperz, 1982) y teorías de los gestos (McNeill, 1985, 1992). Se ha encontrado que los gestos señalan los aspectos centrales de la consigna y podrían, en ese sentido, favorecer la comprensión de los niños de referentes abstractos, vocabulario y las reglas de juego.
\end{abstract}

\section{Introducción}

El objetivo del presente trabajo es el análisis de los gestos que produce una docente de nivel inicial durante la planificación del juego. El estudio de los gestos de la maestra durante la actividad de juego resulta importante debido al impacto que el juego tiene en el desarrollo infantil; en particular cuando "la zona del juego" es andamiada por un otro más experto. En efecto, tanto desde la perspectiva histórico-cultural del desarrollo humano y el aprendizaje (Elkonin, 1980; Vigotsky, 2009) como desde modelos psicolingüísticos actuales (Nelson, 1996; Tomasello, 1998, 2003) se ha mostrado que el juego permite que el niño opere en su zona de desarrollo próximo (Vigotsky, 2009), aprendiendo nuevos conocimientos y desarrollando sus habilidades lingüísticas y discursivas. El lenguaje se desarrolla en la situación de juego pues esta situación de interacción requiere que el niño use sus recursos lingüísticos para comunicarse con otros de modo tal que éstos puedan comprender las intenciones que lo motivan y lleven a cabo sus demandas (Rosemberg, 2008). Asimismo, distintas investigaciones focalizadas en el juego dramático han 
mostrado que éste promueve el uso, por parte de los niños de formas explícitas y descontextualizadas de lenguaje (Pellegrini, 1982, 1984).

Al jugar con otros niños y con adultos, los niños pueden desplegar juegos más complejos en los que tienen la oportunidad de expandir y clarificar sus representaciones de conocimiento (Nelson, 1996, 2007; Rosemberg, 2008). De allí que puedan resultar muy importantes las intervenciones de las maestras para andamiar (Bruner, 1986, 1989) el desempeño de los niños no sólo durante el juego sino también en la interacción previa al mismo. En efecto, en las conversaciones que tienen lugar antes de jugar, la maestra puede colaborar con los niños para que anticipen en qué va a consistir su juego. Estas conversaciones sobre eventos futuros, que adoptan un formato narrativo (Nelson, 1996), se construyen a partir de lo que sucede normalmente, de lo que sucedió en el pasado y de inferencias de lo que podría suceder. Dependen, por lo tanto, de los guiones de conocimiento que los niños puedan activar, con la ayuda de la maestra, para pensar una situación distinta de la actual. Con el andamiaje de la maestra, estos intercambios en los que los niños proyectan un juego que aún no ha ocurrido pueden involucrar planificación y pensamiento hipotético y conducir al niño más allá de la experiencia inmediata. Construir este tipo de representaciones y compartirlas con otros constituye uno de los desarrollos más importantes de los años preescolares (Hudson, 2002, 2006; Nelson, 1996, 2007).

Dado que la elaboración lingüística del juego futuro y su planificación en el marco de la conversación con la maestra puede resultar compleja para los niños y que diversos estudios (Roth, 2001; Valenzeno et al. 2003; Goodrich \& Hudson Kam, 2009) mostraron que los gestos del docente pueden facilitar el aprendizaje de los niños, el objetivo de este trabajo es analizar el uso de gestos por parte de la maestra en la planificación discursiva del juego.

\section{Investigaciones sobre gestos, enseñanza y aprendizaje}

Recientemente, numerosas investigaciones han comenzando a atender sistemáticamente al rol que los gestos cumplen en la comunicación humana (Español, 2004; GoldinMeadow, 2004; Goodrich \& Hudson Kam, 2009; Goodwin, 2000, 2007; McNeill, 1992, 2005; Singer \& Goldin-Meadow, 2005; Valenzeno et al. 2003; Rivière y Sotillo 
1999/2003; Roth, 2001, 2003). Sus resultados señalan que los gestos constituyen un rasgo central del desarrollo, conocimiento, aprendizaje y comunicación humanos a través de las culturas. Se encontró que los gestos son centrales en la cognición (Roth, 2001) y que la información transmitida mediante ellos puede modificar las representaciones mentales de quienes los producen (Beilock \& Goldin-Meadow, 2010).

Una de las principales líneas de investigación sobre los gestos, de corte psicolingüístico, es la desarrollada por McNeill (1985, 1992, 2005), quien restringe el término gesto específicamente a los movimientos de las manos y brazos que encarnan un significado relacionado con el habla al que acompañan. Están referencial y discursivamente orientados y son co-expresivos y sincrónicos con el discurso. (McNeill, 1992; Roth, 2001). Uno de los postulados fuertes de esta corriente, que la distancia de otras, es la afirmación de que los gestos y el discurso comparten la misma estructura psicológica (McNeill, 1985, 1992, 2005). Debido a ello, habla y gesto constituyen modalidades alternativas para expresar un significado. Estas modalidades alternativas son particularmente importantes cuando el hablante presenta "lagunas" en su lexicón (Roth, 2001).

McNeill propuso una clasificación para los gestos en cuatro tipos básicos (McNeill, 1985, 1992), que es la más empleada en el ámbito de la educación (Roth, 2001): los gestos rítmicos, los deícticos, los icónicos y los metafóricos.

Los gestos rítmicos son movimientos simples y rápidos de la mano, vacíos de contenido proposicional, que acompañan a las palabras más importantes de la emisión. Muchas veces señalan el lugar del discurso que el hablante considera importante en relación con el todo (McNeill, 2005).

Los gestos deícticos son señalamientos que pueden ser usados para señalar ya sea en concreto o en abstracto.

Los gestos icónicos abarcan aquellos movimientos de la mano-brazo que guardan una relación perceptual de similitud entre su forma y el contenido semántico de la unidad lingüística a la que acompañan. Es decir, el modo de realización del gesto encarna aspectos retratables del contenido semántico.

Finalmente, los gestos metafóricos son similares a los gestos icónicos en que ellos hacen referencia a una imagen visual; sin embargo, las imágenes a las cuales refieren son 
conceptos abstractos (Roth, 2001; McNeill, 1985). En trabajos más recientes, sin embargo, McNeill prefiere considerar los tipos como dimensiones, en tanto la mayoría de los gestos son multifacéticos - por ejemplo, la iconicidad se puede combinar con la deixis- (McNeill, 2005).

Investigaciones recientes han puesto el foco en la función que los gestos pueden cumplir en la enseñanza y el aprendizaje (Roth, 2001, 2003; Goldin-Meadow, 2004, Singer \& Goldin-Meadow, 2005; Valenzeno, Alibali, y Klatzky, 2003; Goodrich \& Hudson Kam, 2009; Goodwin, 2007). Algunos de estos trabajos recurren a métodos experimentales y otros atiendan al uso de los gestos en situaciones naturales. Entre los primeros pueden mencionarse los trabajos de Goldin-Meadow y colaboradores, quienes se han concentrado en los gestos que niños escolares y docentes producen al explicar y resolver problemas matemáticos. Han hallado que los niños que producen incongruencias entre los gestos y el discurso en la explicación de una tarea - aritmética, en la mayoría de los casos- parecen tener mayor predisposición a resolver correctamente la tarea luego de participar en una situación de enseñanza (Goldin-Meadow, 2004; Roth, 2001; McNeill, 2005). Estos investigadores sostienen que estas incongruencias le permitirían al docente comprender el proceso de formación de nociones en los niños y orientar el proceso de enseñanza de los docentes (Goldin-Meadow, 2004). Asimismo, Goldin-Meadow y colaboradores señalan que en las clases de ciencias y matemáticas, los docentes gestúan con el objeto de clarificar confusiones de los estudiantes. Sin embargo, advierten que aún falta ampliar el conocimiento respecto de cómo los gestos pueden mejorar el desempeño de los alumnos en una tarea específica (Goldin-Meadow, 2004).

En otro trabajo, Singer y Goldin-Meadow (2005) informan los resultados de una serie de experimentos en los que se instruye a niños escolares en problemas de equivalencia matemática en una clase sin la producción de gestos por parte del docente, otra con gestos que acompañan la estrategia de explicación desarrollada verbalmente y una tercera en la que los gestos de la maestra proveen una segunda estrategia de resolución distinta a la provista verbalmente. Los resultados muestran que los niños que mejor resolvieron la tarea fueron los que recibieron una instrucción con gestos que transmitían una segunda estrategia de resolución. 
También cabe señalar dentro de los trabajos experimentales el estudio de Valenzeno et al. (2003), quienes encontraron que los gestos de señalamiento y trazado realizados por docentes en la explicación de la noción de simetría a niños preescolares influyeron positivamente en su desempeño posterior en tareas de aplicación y explicación de dicha noción. Por último, Goodrich y Hudson Kam (2009) observaron en experimentos con adultos y niños de 2, 3 y 4 años que los participantes pueden inferir el significado de formas verbales desconocidas en la interacción a través de los gestos. De ahí su importancia para el aprendizaje del lenguaje.

Entre las investigaciones que atienden al uso de los gestos en contextos naturales de enseñanza cabe mencionar los trabajos de Roth (2001, 2003) y Goodwin (2007). Estos trabajos son de corte etnográfico y conversacional y enfatizan la relación de los gestos con el entorno y las actividades que los participantes están desarrollando. Los trabajos de Roth $(2001,2003)$ se concentraron en clases de física de niños de la escuela secundaria y mostraron que los alumnos, apoyados en gestos y en objetos perceptualmente salientes del entorno, pueden construir explicaciones científicas complejas con una baja carga cognitiva, aún sin dominar el vocabulario de la ciencia en cuestión.

En la misma línea, los trabajos de Goodwin (2000, 2007) atienden a situaciones de aprendizaje de las que participan graduados recientes en arqueología. Ambos trabajos se centran en gestos acoplados al entorno. Se trata de gestos que no pueden ser entendidos por los participantes sin tener en cuenta la estructura del contexto a la que están ligados. El análisis de Goodwin (2007) señala la importancia de la dirección de la mirada de los participantes para construir un gesto como comunicativo; ubicar un gesto dentro de un marco de participación relevante es un modo de establecer públicamente el estatus de una clase particular de gestos. Así considerados, los gestos acoplados al entorno se vuelven centrales en constitución de la mente profesional del arqueólogo en tanto permiten el andamiaje de un experto en las primeras salidas al campo de los novatos para ver las estructuras relevantes en el ambiente (Goodwin, 2007).

Como es posible apreciar en esta revisión de antecedentes, son escasos los trabajos que analizan los gestos de los docentes en su interacción con niños de jardín de infantes y ninguno de ellos emplea metodologías naturalísticas. Por otra parte, la mayoría de los trabajos mencionados fueron realizados con población de nivel socioeconómico 
medio y no se registran investigaciones con grupos urbano marginados como la que constituye el objeto del presente trabajo. Es por ello que se intenta dar cuenta del uso de los gestos por parte de los docentes en situaciones lúdicas en salas de escuelas infantiles a las que asisten niños de poblaciones urbano marginadas. Específicamente se analiza el discurso de la maestra durante la planificación previa al juego. Dado que en ella se representa una situación futura, separada del contexto inmediato, los gestos de la maestra pueden contribuir al andamiaje de la comprensión de dicha tarea.

\section{Corpus y metodología}

Los datos que se analizan en el presente trabajo fueron tomados de un corpus más amplio que constituye la tesis doctoral en curso "La regulación de las situaciones de juego en la educación infantil”1 . La unidad de análisis de la investigación está conformada por situaciones lúdicas observadas durante el momento de "juego en rincones” en escuelas infantiles a las que asisten niños de niños de poblaciones urbano marginadas del conurbano bonaerense de la República Argentina.

El juego en rincones constituye una actividad típica de las escuelas infantiles que configura “una disposición especial y particular del ambiente” (Sarlé, 2001: 70): las salas están organizadas por áreas relacionadas con las actividades que se pueden desarrollar en ellas. Pueden mencionarse como rincones característicos el de la dramatización, donde los niños pueden realizar un juego de roles; el de juegos tranquilos, en el que hay distintos juegos de mesa; el de bloques, donde se ofrecen ladrillos de diversos materiales para realizar construcciones; el de biblioteca y el de arte.

La duración de la actividad oscila entre 45 a 60 minutos y posee tres momentos claramente diferenciados: el de organización, el juego propiamente dicho y el de cierre. En el primero, la maestra anuncia cuáles son los rincones que estarán disponibles para jugar ese día y luego cada niño elige en qué rincón quiere jugar. Eventualmente, la maestra puede recordarles a los niños a qué pueden jugar en cada rincón y luego preguntar, grupal o individualmente, qué es lo que cada niño va a hacer en el rincón, para ayudar a estructurar la actividad. La segunda etapa corresponde al momento de juego propiamente dicho, que tendrá un desarrollo diferente según de qué rincón se trate. El último momento es el de cierre, que comienza cuando la maestra llama a los niños a 
guardar y ordenar los elementos de cada espacio y, en una ronda final, cada niño o grupo cuenta a qué jugó.

Los procedimientos de obtención de información empírica

La metodología usada para obtener los datos fue la observación participante. Las situaciones de juego fueron audio y video grabadas. Las investigadoras que las realizaron eran familiares para la comunidad educativa del jardín ya que la institución participaba desde el año anterior a la toma de datos de un programa de intervención para el desarrollo lingüístico y cognitivo de los niños ${ }^{2}$ llevado a cabo por el mismo equipo de investigación.

Los procedimientos de transcripción y de análisis de la información empírica

Las grabaciones fueron transcritas literalmente para su análisis. Los registros fueron complementados con información del contexto situacional y comportamientos no verbales. Los símbolos utilizados se presentan en el Anexo 1.

El análisis de los datos empíricos adopta una lógica cualitativa que combina el Método Comparativo Constante (Glaser y Strauss, 1967; Strauss y Corbin, 1991) y el empleo heurístico de conceptos del Análisis de la Conversación (Goodwin, 2000, 2007; Sacks, Schegloff y Jefferson, 1974; Tusón, 1995), del microanálisis discursivo de la interacción (Gumperz, 1982) y de los estudios de los gestos (McNeill, 1985, 1992, 2005). Siguiendo el procedimiento de comparación constante, una vez categorizados los gestos, se realizó un análisis comparativo de los incidentes registrados. Este análisis permitió identificar las propiedades que caracterizaban el uso que hacía la maestra de ellos en las secuencias de regulación de la actividad de juego futuro.

Específicamente, en el presente trabajo se analiza un fragmento videograbado de 5 minutos 18 segundos correspondiente a la interacción inicial del momento de juego en rincones entre los niños y la maestra de una sala de 4 años. El hecho de que se trate de una secuencia regulativa (Nussbaum, 2001) hace que haya poca alternancia de turnos entre la maestra y los niños. El segmento transcripto puede dividirse en 3 secuencias: secuencia I (turnos 1 a 29), centrada en las pautas generales de la actividad y el pedido de planificación del juego; secuencia II (turnos 30 a 51), mención de los rincones 
disponibles para jugar ese día; secuencia III (turnos 52 a 85), ajuste de la consigna de planificación en función de los rincones específicos. Asimismo, en dicho fragmento se han registrado un total de 42 gestos por parte de la maestra. Se presenta a continuación un análisis del uso de los mismos en la interacción, atendiendo a cómo en el discurso de la maestra se despliegan y combinan con las intervenciones lingüísticas en las secuencias de regulación.

\section{Secuencia I}

Para analizar el uso de los gestos en este fragmento resulta necesario recuperar el objetivo didáctico general de la secuencia en cuestión. La maestra propone que los niños planifiquen lo que van a hacer en el rincón elegido. Se espera que ello torne la actividad lúdica más compleja y propicia para el aprendizaje de diversas competencias, la lingüística entre ellas. Así por ejemplo, en el caso del juego dramático, se espera que mediante la planificación los niños recuperen más elementos del guión (Nelson, 1996) que van a representar y desplieguen una narrativa más completa. En el caso del juego de construcción, la posibilidad de anticipar qué van a construir les posibilita un mejor dominio del material y ello a su vez puede dar lugar al desarrollo de narraciones en torno a la construcción. En el caso de los juegos de mesa con reglas convencionales, se espera que los niños puedan anticipar aspectos del juego vinculados a las reglas del mismo. En el fragmento analizado, la consigna de planificación de la actividad lúdica se hace explícita en el Ejemplo 2. El ejemplo 1 recupera las pautas generales de la actividad.

\section{Ejemplo $1^{3}$}

Los niños se encuentran sentados en semicírculo frente al pizarrón. La maestra se encuentra frente a ellos, toma unas hojas que tenía en una bolsa y luego les pregunta si saben qué es lo que van a hacer a continuación. Uno de los niños responde que van a jugar "a los rincones".

8. Maestra: M: ((lo observa con cara de sorpresa)) MUY BIE:::N GONZALO vamos a jugar a los rincones pero $\dot{i}\lceil$ podemos jugar en [1TO::DOS] los rincones juntos?

$\left[{ }_{1}\right.$ el BD dibuja un amplio

semicírculo alrededor de su cuerpo] 

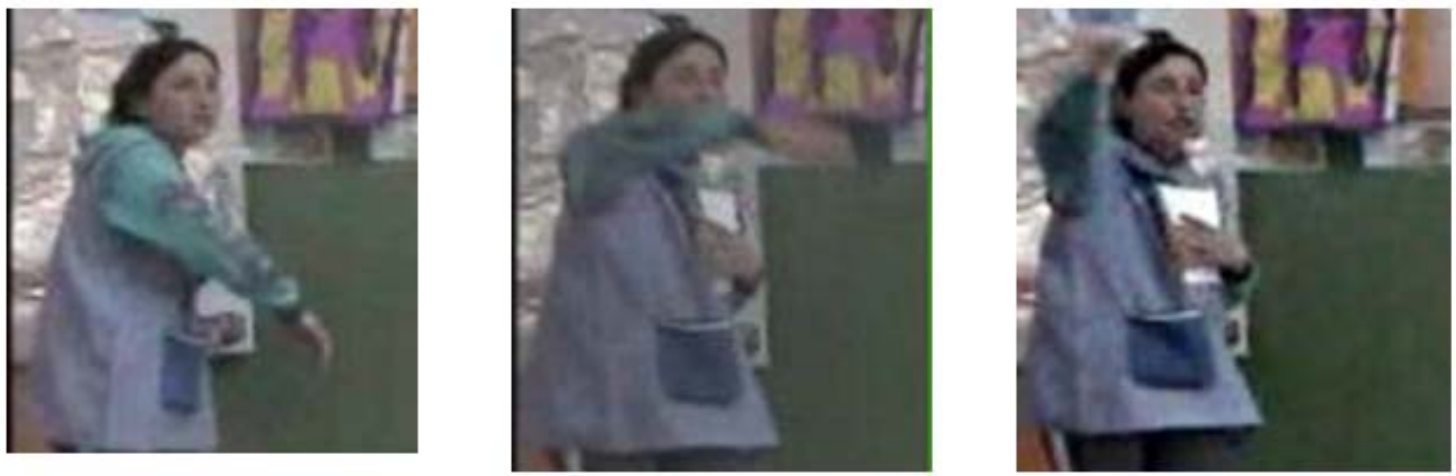

9 Niños: ((responden a coro)) no:::

En el turno 8, los brazos de la maestra dibujan un amplio semicírculo en co-ocurrencia con el ítem léxico - TO::DOS -. Se trata de un gesto metafórico que describe la idea de totalidad. La amplitud de movimiento, junto con el alargamiento vocálico, configuran una pista de contextualización que guía la respuesta posterior de los niños, a coro, en el turno siguiente - no:::-

Ejemplo 2

A continuación, la maestra determina la cantidad de niños que pueden jugar por rincón. Luego establece una pauta general para la actividad, la imposibilidad de cambiar el rincón elegido en la mitad del juego; por lo que invita a los niños a pensar cuidadosamente a qué rincón van a ir a jugar y qué actividad van a desarrollar en el mismo.

25. Maestra: pero [2 YO] (.) si elijo jugar [3en un rinconcito] [2 $M D$ se señala a sí misma] [ [3 BD se abre hacia afuera] no me PUEdo pasar [4 a otro rinconcito]\} [ $\mathrm{B} B \mathrm{D}$ se extiende hacia adelante]
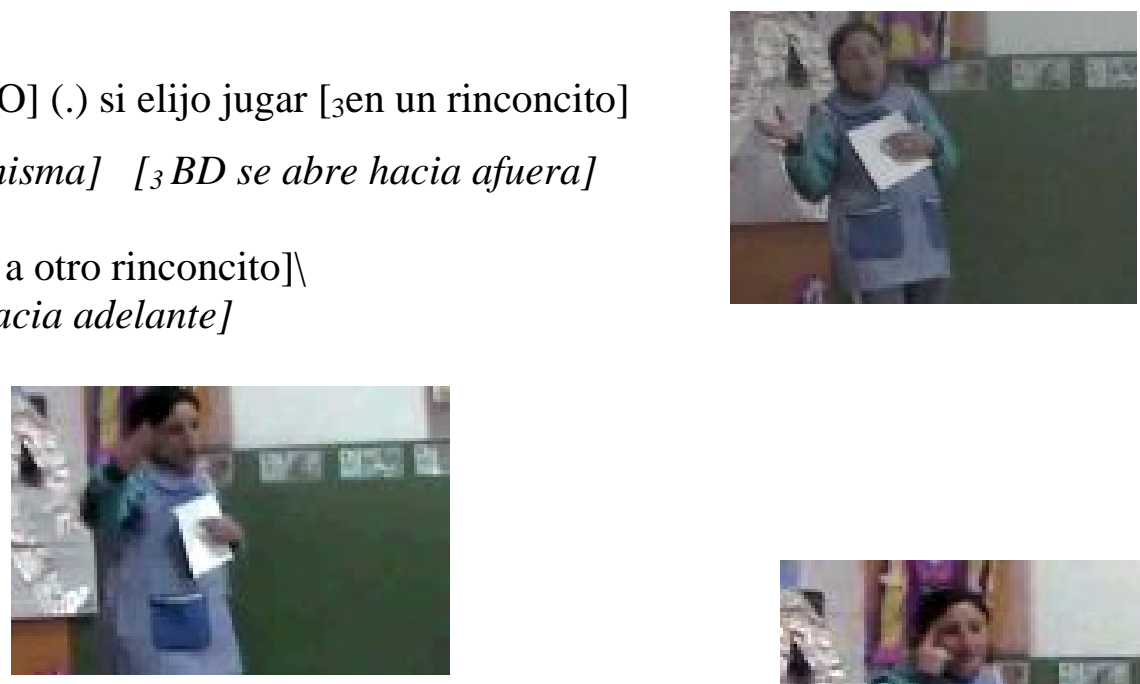

así que tengo que [5 pensar y pensar y pensar pensar muy bien] (.) [5 lleva el dedo índice a la sien]

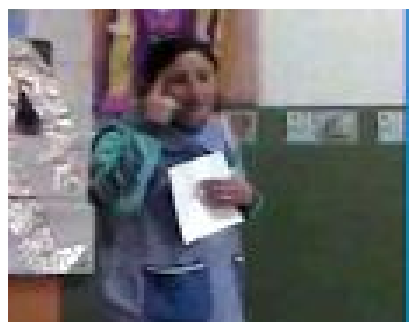


26 NÑO: seño

27 Maestra: a [6qué rincón voy a jugar]

[6 mueve hacia adelante y hacia atrás el dedo índice]

28 Niños: ((hablan en simultáneo))

29. Maestra: y [7 QUÉ voy a hacer en ese =rincón]\=

[7 el dedo índice levantado, movimiento rápido hacia adelante]

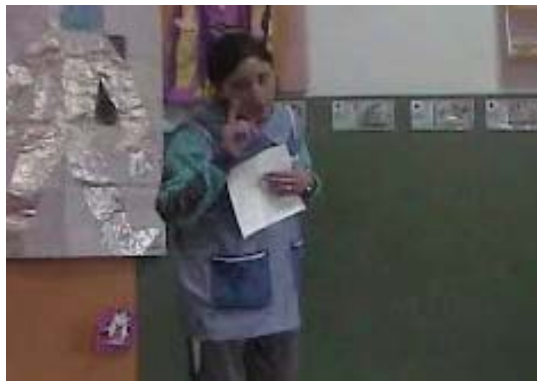

30 Niño : ((Sebastián)) =(www) al de los aU:tos=\ | la caS:Ita $\backslash=$ no seño/= 31 Maestra: =muy bien= muy bien $\backslash$ sebi $\backslash$

En el ejemplo precedente se observan diversos usos de gestos por parte de la docente. En el turno 25, dos gestos deícticos abstractos gestos $n^{\circ} 3$ y 4- ocurren en forma complementaria en la explicación de la regla general de la actividad que establece que los niños, una vez que comienzan a jugar en un rincón, no pueden cambiarse a otro. Estos señalamientos en abstracto refuerzan la oposición entre la elección de "un rinconcito” y “otro rinconcito”, y la imposibilidad del cambio. En función de esta condición, en los turnos 25 y 27 la maestra pide a los niños que piensen muy bien qué rincón van a elegir. En dichos turnos se observa la reiteración del ítem léxico "pensar” junto con el señalamiento de la sien -gesto $\mathrm{n}^{0}$ 5-, de modo tal que la acción de pensar se materializa visualmente para los niños a través del gesto deíctico en el lugar en el que tiene lugar esa acción. Luego, el sentido de pensar se especifica verbalmente en los turnos subsiguientes en las expresiones a qué rincón voy a jugarl y y QUÉ voy a hacer en ese =rincón|=. Estos aspectos de la consigna se focalizan mediante gestos rítmicos -gestos $n^{\circ} 6$ y 7- y entonación enfática. 
La intervención de uno de los niños en el turno 30 alude, precisamente, a los rincones en los que los niños pueden jugar. La maestra aprueba la respuesta del niño, que le da pie a presentar los rincones que estarán disponibles ese día para jugar, dando inicio a la secuencia II.

\section{Secuencia II}

Ejemplo 3

33. Maestra: ((pega un dibujo de una casita con un niño en el pizarrón)) ¿ 「qué rincón es éste? $=$ [8¿ quién se acuerda?] $=$ [ ${ }_{8} M D$ señala el dibujo con la palma hacia arriba] 34. Niño: =el de la casa $=$ 35. Niños: =el de la casita $=$

36. Maestra: muy bien el de la casi::tal

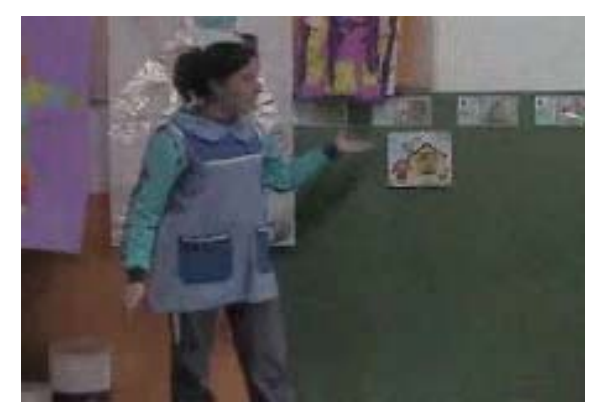

Como puede observarse en el turno 33, la maestra produce un gesto deíctico concreto al señalar con la mano un referente concreto - el dibujo que representa el rincón de dramatizaciones - presente en el contexto de la interacción. Aquí se pone de manifiesto la relevancia de atender a los gestos en el entorno, relevancia señalada por Goodwin (2000, 2007) y Roth (2001, 2003): el gesto deíctico se liga a una imagen que permite que los niños rápidamente identifiquen el rincón introducido. Esta dinámica se reitera en la presentación de todos los rincones, para luego retomar la consigna de planificación en la secuencia III de modo específico para cada rincón.

\section{Secuencia III}

La especificación del sentido del "jugar" en un "hacer" observada en la secuencia I se reitera en otros turnos durante el intercambio en la secuencia III. También en esos segmentos es significativa la presencia de gestos rítmicos, que junto con la reiteración léxica, refuerzan el intento de la maestra de que los niños planifiquen su juego. Sin embargo, el sentido de hacer resulta todavía muy amplio, por lo que entre las intervenciones que produce entre los turnos 76 y 78 la maestra recurre a ejemplos de lo 
que se puede realizar en el rincón de juegos tranquilos y en el de dramatizaciones. Junto con dicha actividad de formulación co-ocurren distintos tipos de gestos que se analizan a continuación.

\section{Ejemplo 4}

76. Maestra: ((se sienta en una silla frente a los niños ) )bueno $\backslash$ (.) voy a sacar $\backslash$ (.) de esta bolsa/ un nombre de un aMIIgo\y ese amigo o amiga/ (.) me va a contar en qué rinconcito va a jugar/ valen escuchá` pero no solo a qué rinconcito va a jugar sino QUÉ va a hacer en ese Lrincón $\backslash$ (.) puedo ir [9por ejemplo al] rinconcito de juegos tranquilos / (.) [9 MD señala el rincón]

((se levanta de la silla y se dirige hacia la estantería en la que están los distintos juegos de mesa)) y puedo ir y tengo ganas (.) 「 por ejemplo/ (.) de armar (.)((toma un juego de encastre y lo muestra a los niños)) ESte rompecabezas de la [10 casita $\backslash$ (.)

que tiene el sol] \se acuerdan que [11 adentro está] el perri::to\

[10 dedos de la MD golpean

pieza del sol]

[11 mueve en círculos el dedo sobre la puerta la de la casa]
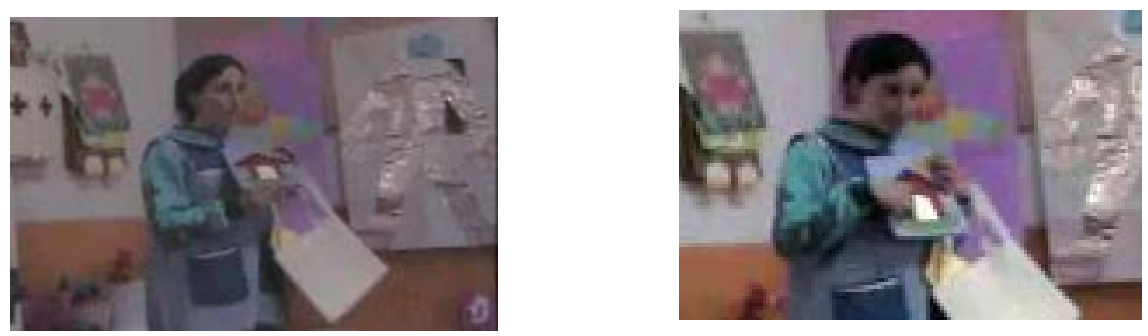

O puedo venir a este rinconcito y TENGO GANAS DE JUGAR (.) al juego de la memoria ((toma un juego de memoria del estante y muestra la caja a los niños)) 77. Niño: $=$ YO tengo ése $=$

78. Maestra: =[12 que era ése= que había que dar vuelta los iguales/ ¿se ]acuerdan/? [12 mueve MD palma arriba, palma hacia abajo, cuatro veces representando el moviendo de descubrir las fichas del juego]
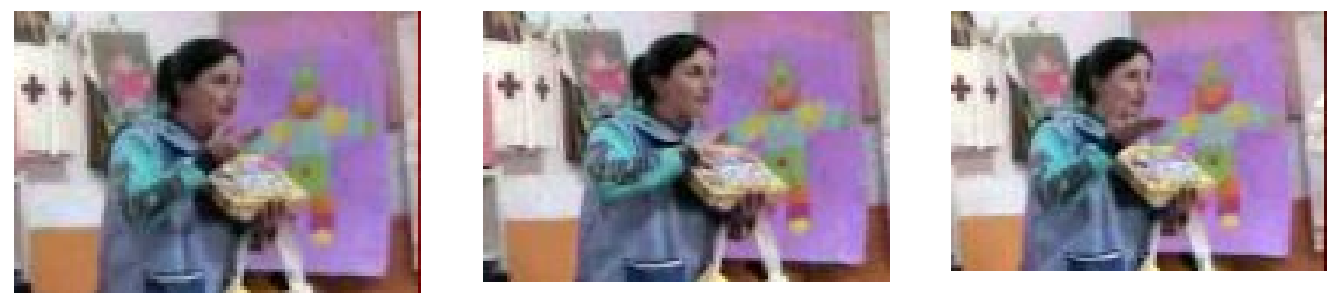

En el inicio del turno 76 la maestra recurre a un señalamiento concreto -gesto $\mathrm{n}^{\circ}$ 9- para iniciar la ejemplificación de la planificación en el rincón de los juegos tranquilos. 
Luego se traslada a dicho sector de la sala y concretiza la actividad que puede desarrollarse allí presentado un juego de encastre: mediante gestos deícticos concretos gestos $\mathrm{n}^{\circ} 10$ y 11- va mencionando sus piezas. De esta forma, precisa rápidamente los referentes de los significados de las palabras. A su vez, el gesto $\mathrm{n}^{0} 11$ es usado para recordarles a los niños un rasgo complejo del encastre en cuestión: una pieza está oculta debajo de otra. El uso del gesto simplifica la explicación y puede tornarla más accesible a los niños.

Luego la maestra toma otro juego del rincón, un juego de memoria, y en el turno 78, la maestra produce un gesto icónico -gesto $\mathrm{n}^{0}$ 12- que representa la estructura profunda del mismo (Sarlé, 2001), a la vez que dice ¿se acuerdan/?: como soporte a la explicación de dar vuelta los iguales, la maestra gira alternadamente la palma de su mano representando el movimiento de descubrir las fichas para hallar las idénticas. La docente se apoya en el formato viso-espacial (McNeill, 1992) del gesto icónico para asegurar la comprensión rápida de la regla básica del memotest. Cabe señalar que el gesto producido cobra sentido a la luz del recuerdo de una experiencia compartida de ese juego.

Posteriormente, en el mismo turno de habla, la maestra continúa con la ejemplificación de lo que se puede hacer en el rincón de dramatizaciones. También allí es marcada la presencia de gestos, como se puede observar en el ejemplo 5.

\section{Ejemplo 5}

78. Maestra: o puedo ir al rinconcito de la caSI:::ta::/ ((se dirige al rincón de la casita)) pero no es que voy a ir al rinconcito de la casita ((se para frente a una alacena de juguete)) y voy a [13 empezar a sacar a tirar todo todo al] piso

[13 mueve el BD hacia arriba y abajo rápidamente tres veces simulando tirar cosas]
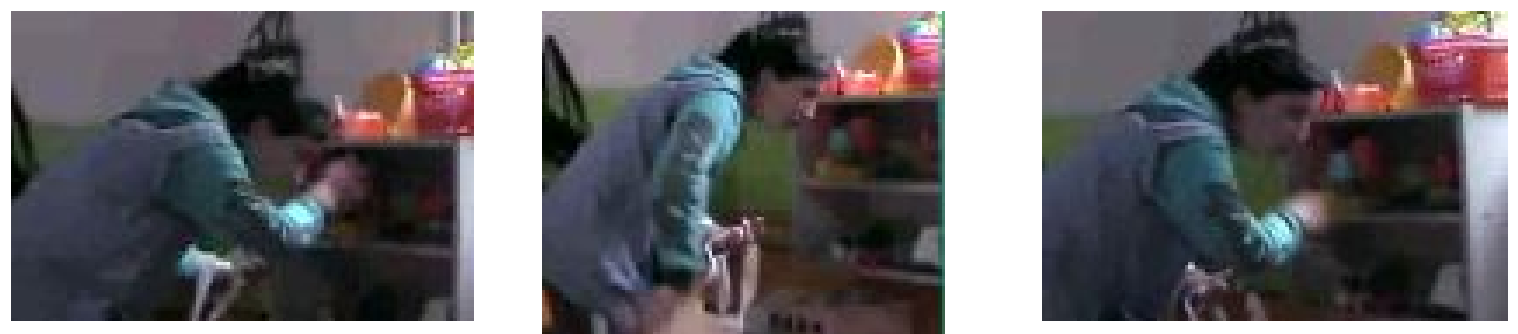

((se dirige al placard y abre y cierra la puerta)) abro el placard saco toda la [14 ropa la tiro] al piso ((los niños ríen))

${ }_{14}$ mueve el BD hacia arriba y abajo rápidamente, simulando tirar cosas] 
Como puede observarse, la maestra grafica mediante gestos icónicos -gestos $n^{0} 13$ y 14- el uso inapropiado de los objetos durante la dramatización: se espera que los niños utilicen los juguetes del rincón con algún fin ligado al guión dramatizado - que, desde la perspectiva vigotskyana, constituye la regla en este tipo de juego (Vigotsky, 1964, 2009)y no que manipulen los materiales sin un objetivo concreto. El gesto icónico hiperbolizado, acompañado de una aceleración en la emisión de las palabras logra el efecto de acentuar lo inapropiado de la conducta que representa, reforzando el objetivo general de la planificación del juego. La risa de los niños pone de manifiesto que han comprendido las intervenciones de su maestra; esto es, se han sentido identificados con los comportamientos que ella señala como inapropiados en el marco de una interacción que no involucra punición.

En el resto del turno78, la maestra ejemplifica lo que se puede hacer en el rincón presentando un posible guión para representar -el de la familia-. Para ello va nombrando los personajes que puede incluir el guión y las posibles acciones que se pueden realizar dentro de los límites que éste impone, como se observa en el ejemplo 6.

\section{Ejemplo 6}

78. Maestra: hoy por ejemplo/ (.) tengo ganas de ser la mamá (.) ((avanza unos pasos hacia los niños con los brazos en posición de jarra)) entonces voy a ser [15 la mamá]/ (.) con la palma extendida]

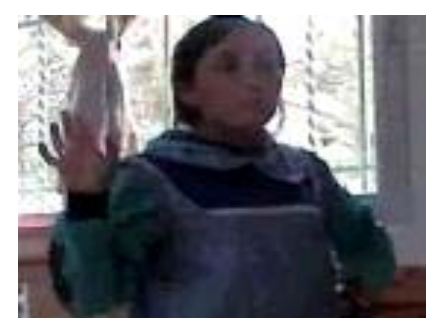

va a haber [16 un papá] / (.) puede haber [17 un hijito] \(.) pueden sacar a pasear al bebé $[16$ camina y extiende el BD hacia adelante] $[17$ mueve lateralmente el BD hacia atrás] 

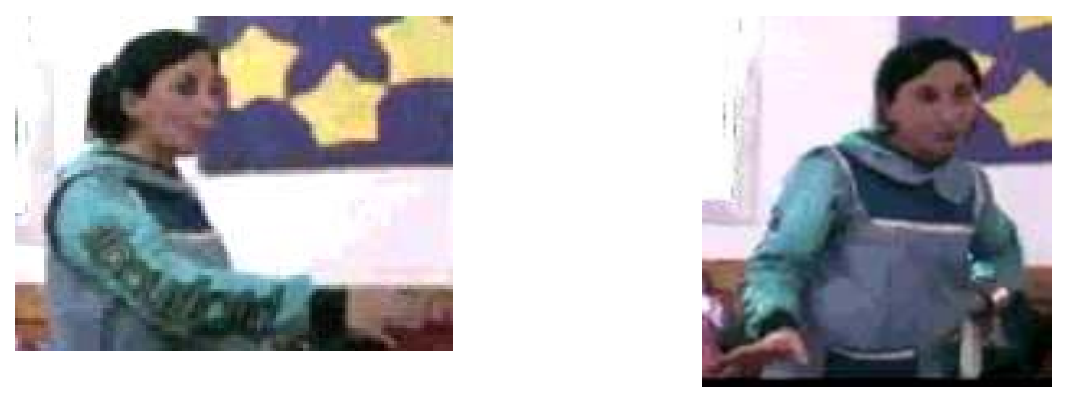

[18 en ese carrito $] \backslash($.) pueden [19 cocinar $\lceil$ aalgo $] \backslash$ (.) pueden hacer un [20 mon「 tón] de cosas (.)

[18 BI señala el carrito] [19 dedos pulgar e índice formando un círculo,

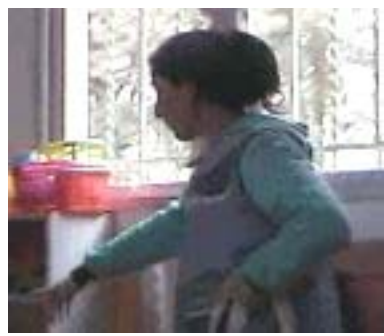
mueve el BD hacia arriba [20 mueve el $B D$ rápidamente y hacia abajo]
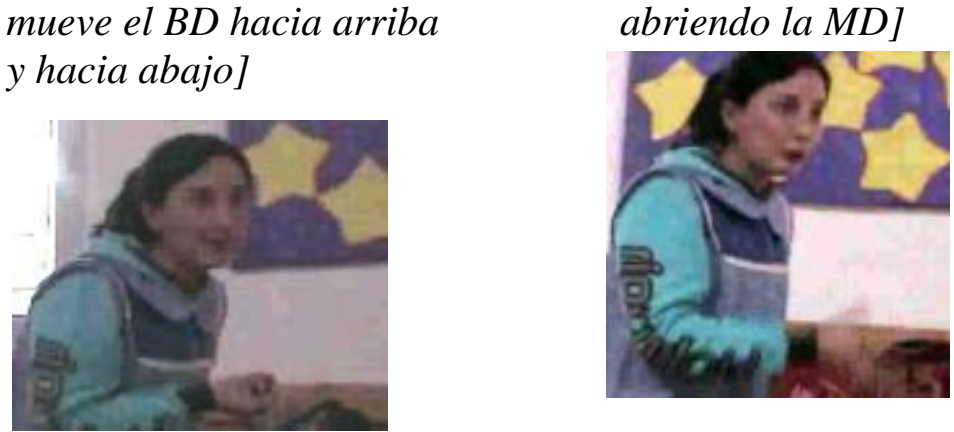

79. Niño: $=$ o mate $=$

80. Maestra: =festejar el= cumpleaños de ALguien (.) tomar mate hacer una torta ir a comprar\ (.)

81. Niños: (www)

82. Maestra: un montón de cosas\

La mención de cada componente es reforzada por gestos rítmicos -gestos $n^{0} 15,16,17$, 19 y 20- y deícticos -gesto $\mathrm{n}^{0}$ 18- que en función de la posición del brazo y de la mano delimitan distintos actores y objetos en el juego. De esta forma se aclara en qué consiste el hacer en el rincón de la casita; hecho que se pone de manifiesto en las intervenciones de los niños que mencionan aspectos del guión, como se observa en el turno79 =o mate $=$.

\section{Conclusión}

En el presente trabajo se identificaron diversos usos de los gestos en la planificación del juego en rincones, así como también formas diversas en las que los gestos se integran en correspondencia con las intervenciones verbales en la situación de enseñanza. El análisis 
realizado puso de manifiesto cómo los gestos pueden contribuir a focalizar la atención de los niños en los aspectos centrales de la consigna, a precisar y a destacar los componentes de un guión al que los niños podrán recurrir para organizar su juego, a la comprensión de las reglas de juego; permiten ejemplificar y concretizar los conceptos abstractos que configuran el discurso. Tal como ha sido señalado en otras investigaciones con niños de mayor edad, estos gestos que acompañan las intervenciones verbales de las maestras en las situaciones de enseñanza pueden tener un impacto en el aprendizaje de los niños (Gaythwaite, 2005; Roth, 2001; Singer y Goldin-Meadow, 2005). Los resultados del presente análisis muestran, asimismo, que las intervenciones de la maestra se acompañan no sólo de gestos deícticos e icónicos sino también de gestos rítmicos; gestos que, hasta el presente, no han sido tratados en detalle en las investigaciones que atienden al uso de los gestos en las situaciones de enseñanza.

Considerar el momento de la planificación del juego resulta fundamental en términos del andamiaje para la futura actividad lúdica. Constituye, asimismo, una oportunidad para promover en los niños habilidades para construir representaciones lingüísticas de eventos anticipados y para planificar. En efecto, atender a los gestos que la maestra realiza durante la planificación permitió dar cuenta del anclaje que hace posible que los niños sostengan la interacción acerca de un hecho futuro. En estas conversaciones previas al juego propiamente dicho la maestra tiene la oportunidad de colaborar con los niños para que proyecten sus acciones en el futuro. A través de diferentes formas, tanto verbales como no verbales, les muestra a los niños las distintas alternativas y el carácter hipotético que puede adoptar la acción eventual. Como lo señalaron Nelson $(1996,2007)$ y Hudson $(2002,2006)$ pensar y conversar sobre el futuro les permite a los niños organizar las acciones y entender cómo éstas se relacionan con un objetivo, representarse una situación distinta de la actual y pensar en lo que va suceder, es decir, proyectarse en el futuro.

\section{Referencias bibliográficas}

Beilock, S. L \& S. Goldin-Meadow (2010). Gesture changes thought by grounding it in Action. Psychological Science, 21, 11, 1605-1610.

Bruner, J. (1986). El habla del niño. Barcelona: Paidós. 
Bruner, J. (1989). Acción, pensamiento y lenguaje, Comp. J. Linaza. Madrid: Alianza Psicología.

Elkonin, D. (1980). Psicología del juego. Madrid: Visor Aprendizaje.

Español, S. (2004). Cómo hacer cosas sin palabras. Gesto y ficción en la infancia temprana. Madrid: Antonio Machado.

Gaythwaite, S. E. (2005). Didn't you see what I meant? Informing gestures in teaching and learning. Curriculum and Teaching Dialogue, 7, 97-108.

Glaser, B. \& Strauss, A. (1967). The discovery of grounded theory. Chicago: Aldine Publishing Company.

Goldin-Meadow, S. (2004) Gesture's role in the learning process. Theory into practice. College of Education, The Ohio State University, 43, 4,

Goodrich, W. \& C. L. Hudson Kam (2009). Co-speech gesture as input in verb learning. Developmental Science, 12, 1, 81-87.

Goodwin, C. (2000). Action and embodiment within situated human interaction. En Journal of Pragmatics, 32, 1489-1522.

Goodwin, C. (2007). Enviroment coupled gestures. En S. Duncan, J. Cassell, y E. Levy (Eds.) Gesture and the Dinamic Dimension of Language. Amsterdam/Philadelphia: John Benjamins Publishing Company.

Gumperz, J. (1982). The linguistic bases of communicative competence. En D. Tannen (Ed.) Analyzing discourse: text and talk. Washington: Geogetown University Press.

Hudson, J. A. (2002). “Do you know what we're going to do this summer?”: Mothers' talk to preschool children about future events. Journal of Cognition and Development, 3(1), 49-71.

Hudson, J. A. (2006). The development of future time concepts through mother-child conversation. Merrill-Palmer Quarterly, 52, 70-95.

McNeill, D. (1985) So you think gestures are nonverbal? Psychological Review, 92, 3, 350-371.

McNeill, D. (1992). Hand and mind: What gesture reveals about thought. Chicago, IL: University of Chicago Press.

McNeill, D. (2005). Gesture and thought. Chicago: University of Chicago Press.

Nelson, K. (1996). Language in cognitive development. The emergence of the mediated mind. Nueva York: Cambridge University Press.

Nelson, K. (2007). Young minds in social worlds: Experience, meaning, and memory. Cambridge, MA: Harvard University Press.

Nussbaum, L. (2001). El discurso en el aula de lenguas extranjeras. En L. Nussbaum, y M. Bernaus, (Eds.) Didáctica de la lengua extranjera en la ESO. Madrid: Síntesis.

Pellegrini, A. (1982). The construction of cohesive text by Preschoolers in two play contexts. Discourse Processes, 5, 101-108.

Pellegrini, A. (1984). Effects of experimental play contexts of the development of preschooler's functional language. Journal of Pragmatics, 8, 211-219.

Rivière, Á. y Sotillo, M. (1999/2003). Comunicación, suspensión y semiosis humana: Los orígenes de la práctica y de la comprensión interpersonales. En M. Belinchón, A. Rosa, M. Sotillo \& I. Marichalar (Comp.) Ángel Rivière. Obras Escogidas, Vol III, . 181-201. Madrid: Panamericana. 
Rosemberg, C. (2008). El lenguaje y el juego en la educación infantil. En Sarlé, P. (coord.) Enseñar en clave de juego. Enlazando juegos y contenidos. Buenos Aires: Novedades Educativas.

Roth, W-M. (2001). Gestures: Their role in teaching and learning. Review of Educational Research, 17, 3, 365-392.

Roth, W-M. (2003). Gesture-speech phenomena, learning, and development. Educational Psychologist, 38, (4), 249-263

Sacks, H. Schegloff, E. A. y G. Jefferson (1974). A simplest systematic for the organization of turn-taking for conversation. Language, $\mathrm{N}^{\circ} 50,696-735$.

Sarlé, P. (2001). Juego y aprendizaje escolar: los rasgos del juego en la educación infantil. Buenos Aires: Novedades Educativas.

Singer, M. A. y S. Goldin-Meadow (2005) Children learn when their teacher's gestures and speech differ. Psychological Science, 16, 2, 85-89.

Strauss, A. L., Corbin, J. (1991). Basics of qualitative research. Newbury Park - London - New Delhi: Sage.

Tomasello, M. (1998). The new psychology of language: Cognitive and functional approaches to language structure. Mahwah, NJ: Lawrence Erlbaum Associates.

Tomasello, M. (2003). Constructing a language: A usage-based theory of language acquisition. Massachusetts: Harvard University Press.

Tusón, A. (1995). Análisis de la conversación. Barcelona: Ariel.

Valenzeno, L., Alibali, M. \& R. Klatzky (2003).Teachers' gestures facilitate students learning: A lesson in symmetry. Contemporary Educational Psychology , 28, 187-204.

Vigotsky, L. S. (1964). Pensamiento y lenguaje. Buenos Aires: Editorial Lautaro.

Vigotsky, L. S. (2009) El desarrollo de los procesos psicológicos superiores. Buenos Aires: Crítica.

\section{Anexo 1: Glosario de símbolos de transcripción utilizados}

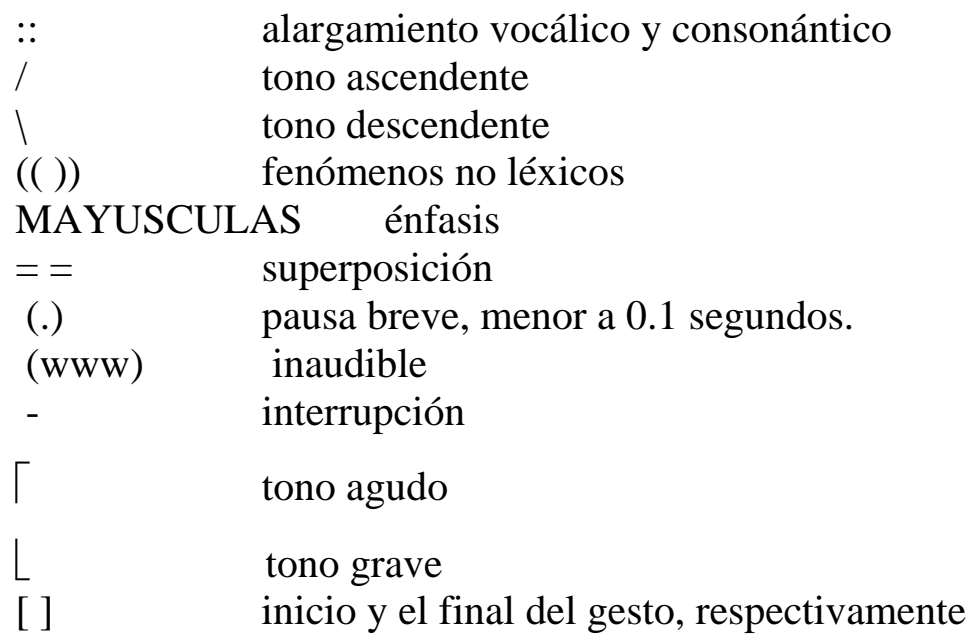

Palabras en itálica descripción del gesto

MD mano derecha

MI mano izquierda 


$\begin{array}{ll}B D & \text { brazo derecho } \\ B I & \text { brazo izquierdo }\end{array}$

${ }^{1}$ Los datos de la tesis doctoral fueron tomados en el marco del "Programa Oscarcito. Desarrollo Lingüístico y Cognitivo en la infancia” dirigido por la Dra. Celia Renata Rosemberg y Ana María Borzone y cuenta con el apoyo del CONICET- CIIPME, la Fundación Care de Alemania y la Fundación Árcor de Argentina. La investigación se desarrolla asimismo en el marco del UBACYT "El lenguaje, el juego y la Educación Infantil” dirigido por C. R. Rosemberg.

${ }^{2}$ Ver supra.

${ }^{3}$ Las autoras tienen el permiso de la maestra para publicar sus imágenes.

\section{Referencias de las autoras:}

Maia Julieta Migdalek es licenciada en Letras por la Universidad de Buenos Aires. Actualmente cursa la maestría en Análisis del Discurso y el Doctorado en Educación, ambos en la Universidad de Buenos Aires, con una beca doctoral del Consejo Nacional de Investigaciones Científicas y Técnicas en Argentina (CONICET).

Email: maiamig@hotmail.com

Celia Renata Rosemberg es investigadora independiente del Consejo Nacional de Investigaciones Científicas y Técnicas en Argentina (CONICET) y Profesora Adjunta concursada en la Universidad de Buenos Aires.

Para citar este artículo:

Migdalek, M-J. \& Rosemberg, C. (2012) El uso de los gestos en el discurso docente durante la planificación del juego en el jardín de infantes. Bellaterra Journal of Teaching \& Learning Language \& Literature, 5(3), 25-43. 